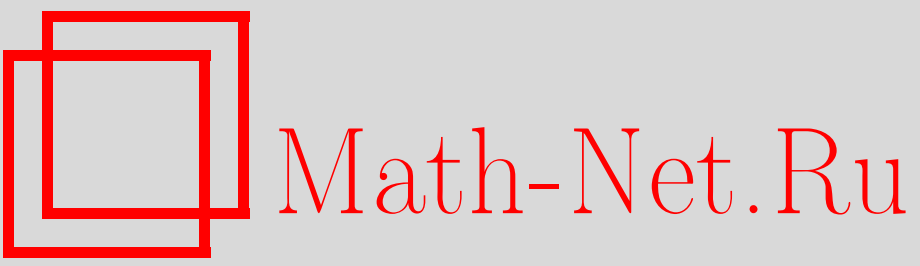

Ж. Бургейн, Недавний прогресс в квазипериодических решеточных операторах Шрёдингера и гамильтоновых дифференциальных уравнениях с частными производными, УМH, 2004, том 59, выпуск 2, 37-52

DOI: https://doi.org/10.4213/rm716

Использование Общероссийского математического портала Math-Net.Ru подразумевает, что вы прочитали и согласны с пользовательским соглашением

http: //www.mathnet.ru/rus/agreement

Параметры загрузки:

IP : 34.239 .49 .27

26 апреля 2023 г., 09:17:39 


\title{
НЕДАВНИЙ ПРОГРЕСС В КВАЗИПЕРИОДИЧЕСКИХ РЕШЕТОЧНЫХ ОПЕРАТОРАХ ШРЁДИНГЕРА И ГАМИЛЬТОНОВЫХ ДИФФЕРЕНЦИАЛЬНЫХ УРАВНЕНИЯХ С ЧАСТНЫМИ ПРОИЗВОДНЫМИ
}

\author{
Ж. БУРГЕЙн
}

\begin{abstract}
В данной работе представлен обзор последних исследований по квази-периодическим локализациям на решетках (как методов основанных на теории возмущений, так и непертурбативных методов) и приложений KAM теорий применительно к бесконечномерньм гамильтоновым системам. Особое внимание уделяется приложениям этих исследований применителшно к уравнению Шредингера и волновому уравнению с периодичными граничными условиями, а также применительно к нелинейному случайному уравнению Шрёдингера с короткодействующими потенциалами.

Библиография: 54 названия.
\end{abstract}

\section{СОДЕРЖАНИЕ}

Введение

1. Решеточные операторы Шрёдингера с квазипериодическим потенци-

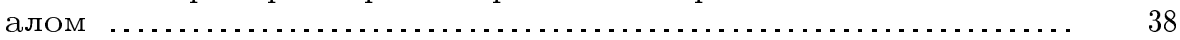

2. Решеточные операторы Шрёдингера, связанные с косым сдвигом .... 43

3. KAМ-теория в гамильтоновых дифференциальных уравнениях с частными производными и бесконечномерных системах .............. 45

Список литературы .............................................. 50

\section{Введение}

Цель доклада - описать некоторые “недавние” достижения в общей области квазипериодической локализации для решеточных операторов Шрёдингера и теорий типа KAM для гамильтоновых дифференциальных уравнений с частными производными (таких, как нелинейные уравнения Шрёдингера и нелинейные волновые уравнения). Это - область продолжающихся исследований, и наше изложение будет сфокусировано лишш на нескольких аспектах этой темы, главным образом тех, в развитии которых участвовал автор. Мы, конечно, предпринимаем здесь небольшую попытку представить полный отчет об исторических истоках, которая, однако, затруднена ограниченным объемом статьи. Бо́льшая часть рассматриваемых здесь тем уходит корнями в плодотворную работу Колмогорова об устойчивости квазипериодического движения 
при малых возмушениях [42] или тесно связана с ней. Таким образом, вот некоторые из обсуждаемых ниже тем:

(i) спектр и собственные функции решеточных операторов Шрёдингера с квазипериодическим потенциалом (локализации Андерсона и т. д.);

(ii) линейные уравнения Шрёдингера с зависящим от времени потенциалом, в частности, квантовьй сингулярно возмущенньй ротатор;

(iii) KAM-торы (квази- и почти периодические решения) гамильтоновых дифференциальных уравнений с частными производньми.

Список литературы обслуживает только эту задачу и даже в этом виде очень неполон. Его единственная цель - связать приведенные ниже утверждения с той или иной существуюшей литературой. Имеются и другие обзоры, посвяшенные операторам Шрёдингера (см., например, [51]).

\section{1. Решеточные операторы Шрёдингера с квазипериодическим потенциалом}

Рассмотрим решетку $\mathbb{Z}^{d}, d \geqslant 1$, и пусть $\Delta$ - обьчньй решеточный лапласиан:

$$
\Delta\left(n, n^{\prime}\right)= \begin{cases}1, & \text { если } \sum\left|n_{j}-n_{j}^{\prime}\right|=1, \\ 0 & \text { в противном случае. }\end{cases}
$$

Во многих случаях аналогичные результаты могут быть получены, если мы заменим $\Delta$ матрищей тёплицева типа $S\left(n, n^{\prime}\right)=S\left(n-n^{\prime}\right)$, где $S(n) \rightarrow 0$ достаточно быстро при $|n| \rightarrow \infty$.

При данном потенщиале $V=\left(V_{n}\right)_{n \in \mathbb{Z}^{d}}$ основная задача состоит в том, чтобыпонять природу спектра и поведение собственных функций оператора

$$
H=\Delta+\lambda V
$$

Две модели, особенно интересные для математической физики (и восходящие к работам Андерсона, Пайерлса, Хофштадтера и др.), - случай квазипериодического потенциала $V$ и случай стохастического потенциала $V$ (т.е. все $V_{n}-$ независимые случайные величины).

Обе модели неплохо изучены при $d=1$ и гораздо хуже при $d>1$. Здесь мы занимаемся только квазипериодической моделью. Начиная с плодотворных работ Синая и его сотрудников (см. [52], [30] и т. д.) и Фрёлиха-Спенсера-Виттвера [35], в последние годы было достигнуто много успехов. Мы опишем ниже некоторые новые результаты. Как станет ясно, они вносят свой вклад в более полную картину этой области знаний.

"Нововведения" есть и на методологическом уровне. В то время как первоначально единственньй подход был основан на теории возмушений и использовал методы, связанные с собственными значениями, мы располагаем теперь подходом, не используюшим теорию возмущений, а также мошной технологией теории возмущений (основанной на методах теории субгармонических функций и теории полуалгебраических множеств) для изучения многомерных задач.

(i) Размерность 1 и одна частота. В этой постановке наиболее тщательно изученная задача - это, конечно, так назьваемьй "почти оператор Матье"

$$
H_{\lambda}=\lambda \cos (\theta+n \omega)+\Delta
$$


возникаюший, в частности, в контексте блоховских электронов в магнитном поле.

Мы будем говорить, что $H_{\lambda}$ удовлетворяет локализации Андерсона (ЛА), если существует чистая точка (ч.т.) спектра с экспоненциально локализованными собственными функциями. Первые результаты, относяшиеся к (1.3), принадлежат Синаю [52] и Фрёлиху-Спенсеру-Виттверу [35], которые установили для больших $\lambda$ наличие локализации Андерсона для большинства пар $(\omega, \theta)$ (более точно, $\omega$ предполагается диофантовым, а $\theta$ берется вне малого множества в смысле меры). Оба доказательства в [52] и [35], а также более поздние работы, подобные [31], используют методы теории возмущений (без явного уточнения, насколько велико $\lambda$ ) и сильно зависят от рассуждений о собственных значениях, используюших методы теории возмушений (включая возмущения второго порядка). Значительньй успех был достигнут в работе Житомирской [41], в которой локализация Андерсона была установлена при $\lambda>2$. Подход в [41] существенно отличается от подхода работ [52] и [35] в том смысле, что он не использует методы теории возмушений. Таким образом, мы предположим, что $\omega-$ диофантово, а $\theta$ находится вне некоторого множества меры нуль, но условие $\lambda>2$ не зависит от специфических диофантовых условий, наложенных на $\omega$. Что касается техники, работа [41] не использует параметризацию собственных значений, а функция Грина оценивается непосредственно с помошњю правила Крамера.

Спектральная картина задачи (1.3) имеет следуюший вид.

Для почти всех $(\theta, \omega)$ (известны и много более точные утверждения)

$$
\left\{\begin{array}{l}
\lambda>2: H_{\lambda} \text { имеет чисто точечньй спектр с ЛА, } \\
\lambda=2: H_{\lambda} \text { имеет чистьй сингулярньй спектр, } \\
\lambda<2: H_{\lambda} \text { имеет чистьй абсолютно непрерьвньй спектр. }
\end{array}\right.
$$

Таким образом, при $\lambda=2$ имеем фазовый переход (см. [37], [46]). Модель (1.2) имеет то специальное свойство, что двойственность Обри (по существу - преобразование Фурье [2]) меняет местами модели $H_{\lambda}$ и $H_{\frac{4}{\lambda}}$. При других потенциалах разумно ожидать возможное совместное существование нескольких спектральных типов.

В отличие от [52] и [35], доказательство в [41] существенно использует специальную структуру задачи (1.2), и, к сожалению, замена потенциала $v(\theta)=\cos \theta$ другим тригонометрическим полиномом делает часть доказательства неверной. Более мощное доказательство было развито в [16].

ТЕОРема 1.5. Пусть $v$-вещественно-аналитическая функиия на $\mathbb{T}=\mathbb{R} / \mathbb{Z}, u$ nусть $H_{\omega}=v(n \omega) \delta_{n n^{\prime}}+\Delta$. Пусть $\left[E_{1}, E_{2}\right] \subset \mathbb{R}-$ интеграл энергии. Предположим, что для всех или почти всех $\omega$ показатель Ляпунова $L(E)$ положстелен при всех $E \in\left[E_{1}, E_{2}\right]$. Тогда для почти всех $\omega$ гамильтониан $H_{\omega}$ удовлетворяет условию ЛА на $\left[E_{1}, E_{2}\right]$.

Напомним, что

где

$$
L(E)=\lim _{N \rightarrow \infty} \frac{1}{N} \int \log \left\|M_{N}(E, \theta)\right\| d \theta
$$

$$
M_{N}(E, \theta)=\prod_{N}^{1}\left(\begin{array}{cc}
-v(\theta+n \omega)+E & -1 \\
1 & 0
\end{array}\right)
$$

- трансфер-матрица. Действительно, уравнение $H \xi=E \xi$ можно переписать в виде

$$
\left(\begin{array}{c}
\xi_{N} \\
\xi_{N-1}
\end{array}\right)=M_{N}\left(\begin{array}{l}
\xi_{1} \\
\xi_{0}
\end{array}\right)
$$


Мы также напомним, что, согласно результату Хермана [39] и Сореца-Спенсера [54], для любого данного не равного константе вещественно-аналитического потенциала $v$ на $\mathbb{T}$, полагая $H=\lambda v(\theta+n \omega) \delta_{n n^{\prime}}+\Delta$, получаем

$$
L(E)>\frac{1}{2} \log \lambda \text { при } \lambda>\lambda_{0}(v)
$$

(независимо от числа врашения $\omega$ ). Объединяя этот факт с теоремой 1.5, получаем следующий результат.

ТеОРема 1.7. Пусть $v$ вещественно аналитичен и не постоянен на $\mathbb{T}=\mathbb{R} / \mathbb{Z}$. Тогда при $\lambda>\lambda_{0}(v)$ гамильтониан $H=\lambda v(n \omega+\theta) \delta_{n n^{\prime}}+\Delta$ проявляет ЛА для почти всех $\omega, \theta \in \mathbb{T}$.

Как и в [41], ключевую роль в [16] играют оценки, содержашие показатели Ляпунова (подобнье оценкам больших уклонений для $\frac{1}{N} \log \left\|M_{N}(E, \theta)\right\|$, получаемьм из теории субгармонических функций). Важная новая составная часть в доказательстве теоремы 1.5 (и во всех последуюших результатах, связанных с обсуждаемыми здесь квазипериодическими гамильтонианами) - теория полуалгебраических множеств. Следует заметить, что теорема 1.5 приводит к несколько более слабому утверждению, чем [41], в том смысле, что не дает арифметического описания хороших частот.

Снова используя двойственность Обри, получаем следующую часть абсолютно непрерьвного спектра.

Tеорема 1.8 [19]. Пусть $H=\lambda v(n \omega+\theta) \delta_{n n^{\prime}}+\Delta$, zде v вещественно аналитичен на $\mathbb{T}$, а $\omega$ диофантово. Тогда при $\lambda<\lambda_{0}(v)$ гамильтониан $Н$ имеет чистый абсолютно непрерывный спектр.

Вернемся к теореме 1.5. В дальнейшем было доказано [36], в тех же условиях и в предположении, что

$$
L(E)>\delta>0 \text { при } E \in\left[E_{1}, E_{2}\right],
$$

что суммарная плотность состояний $(\mathrm{C \Pi C}) k(E)$ непрерьвна по Гёльдеру на $\left[E_{1}, E_{2}\right]$ :

$$
\left|k(E)-k\left(E^{\prime}\right)\right|<C\left|E-E^{\prime}\right|^{\rho} \text { при } E, E^{\prime} \in\left[E_{1}, E_{2}\right]
$$

(если $\omega$ удовлетворяет сильному диофантову условию).

Дальнейшие уточнения (см. [4]) показывают, что на самом деле можно выбрать $\rho=$ $\rho(v)>0$ независимо от $\delta$ в (1.9), и, таким образом, $\rho$ зависит только от границ для $v$, но постоянная $C=C(\delta)$ в (1.10) действительно зависит от $\delta$.

В случае "почти оператора Матье" $H=\lambda(\cos (\theta+n \omega)) \delta_{n n^{\prime}}+\Delta$ имеем (при $\left.\omega \notin \mathbb{Q}\right)$ (cM. $[20])$

$$
L(E)=\max \left(0, \log \frac{|\lambda|}{2}\right), \text { если } E \in \operatorname{Spec} H .
$$

Работа Хелффера-Сьёстранда [38] показьвет, что если $\lambda=2$ и $\omega$ имеет такое равномерно большое разложение в непрерывную дробь $\left\{a_{n}\right\}$, что

$$
\varlimsup \frac{1}{N} \sum_{1}^{N} a_{n}=\infty,
$$

то гёльдерова регулярность СПС не имеет места. 
Наконец, работы [37] и [38] показьвают, что при $\lambda=2$ "почти оператор Матье" имеет канторов спектр. В последнее время этот факт был доказан для любого $\lambda \neq 0$ Пюигом [49].

(ii) Размерность 1 и несколько частот. С ледуюшие дальнейшие результаты, касающиеся проблемы локализации в случае многих частот, были получены в [16], [4].

Теорема 1.12. Аналог теоремы 1.7 выполняется для вещественно-аналитических потенциалов $v$ на $\mathbb{T}^{d}$ и для $H_{\omega}=v(n \omega) \delta_{n n^{\prime}}+\Delta\left(n \in \mathbb{Z}, \omega \in \mathbb{T}^{d}\right)$.

Что касается положительности показателя Ляпунова, то имеется следующий полньй аналог теоремы Сореца-Спенсера для нескольких переменных (но с существенно более сложным доказательством); см. [8], [10].

ТЕОРема 1.13. Пусть $v$ - не постоянный вещественно-аналитический потенциал на $\mathbb{T}^{d}$, и пусть $H=\lambda v(n \omega+\theta) \delta_{n n^{\prime}}+\Delta$, как и выше. Тогда при $|\lambda|>\lambda_{0}(v)$ имеем

$$
L(E)=\frac{1}{2} \log |\lambda| \quad \text { для всех } \omega \text { и } E .
$$

Отсюда следует

Теорема 1.14. Имеет место аналог теоремы 1.7 в одномерном случае мноzux частот.

Вариант этой теоремы в теории возмущений был дан в [28].

Рассмотрим теперь вопрос об абсолютно непрерьвном спектре в случае многих частот. Имеется следуюшая теорема, используюшая методы теории возмушений.

Teоpema $1.15[27],[26]$. Пусть $H=\lambda v(n \omega+\theta) \delta_{n n^{\prime}}+\Delta\left(n \in \mathbb{Z}, \omega \in \mathbb{T}^{d}\right)$, əде v не постоянен и вещественно аналитичен на $\mathbb{T}^{d}$. Тогда при $\lambda<\lambda_{0}(v, \varepsilon)$ и $\omega \notin \Omega$, где mes $\Omega<\varepsilon$, гамильтониан $H_{\omega}$ имеет чистый абсолютно непрерывный спектр.

Поразительно, что утверждение не используюшей методов теории возмушений теоремы 1.5 нарушается в случае многих частот.

Теорема 1.16 [5; ч. II]. Пусть $v$ - тригонометрический многочлен на $\mathbb{T}^{2} c$ невырожденным максимумом. Тогда при $\omega \in \mathbb{T}^{2}$, пробегающем множество положительной меры, гамильтониан $H_{\omega}=v\left(n \omega_{1}, n \omega_{2}\right) \delta_{n n^{\prime}}+\Delta$ имеет точечный спектр $P$, mes $\bar{P}>0$, с әкспоненииально локализованныцми состояниями.

(iii) Квазипериодические модели при $D>1$. Рассмотрим теперь решеточные операторы Шрёдингера на $\mathbb{Z}^{d}, d \geqslant 2$.

Частньй, но важньй случай имеет вид

$$
H=\lambda v(n \cdot \omega)+\Delta,
$$

где $v$ не постоянен и вещественно аналитичен на $\mathbb{T}, \omega \in \mathbb{T}^{d}$ и $n \in \mathbb{Z}^{d}$ ( $\Delta$ определен формулой (1.1)). Есть следуюший результат о локализации, используюший методы теории возмущений (см. [27], [4]). 
Tеорема 1.18. При данном $\varepsilon>0$ есть такое $\lambda_{0}=\lambda_{0}(v, \varepsilon)$, ито при $\lambda>\lambda_{0} u$ $\omega \notin \Omega$, где mes $\Omega<\varepsilon\left(\Omega \subset \mathbb{T}^{d}\right.$ зависит от $\left.\lambda v\right)$, гамильтониан $H$, определенный формулой (1.17), удовлетворяет ЛА.

Вариант этого результата, не использующий методов теории возмущений (т.е. $\lambda>$ $\lambda_{0}(v), \omega$ лежит вне некоторого множества меры нуль), неверен. Если в теореме 1.16 вьполняется условие

$$
v\left(\theta_{1}, \theta_{2}\right)=\alpha\left(\cos \theta_{1}+\cos \theta_{2}\right) \quad(\alpha>0 \text { мало })
$$

то из двойственности Обри на самом деле следует, что гамильтониан (1.17), определенный формулой

$$
H=\frac{4}{\alpha} \cos (n . \omega)+\Delta,
$$

имеет собственные продолженные состояния (для $\omega$, пробегаюшего множество положительной меры).

ТеОРема 1.19 [18]. Пусть $d=2$, и пусть $v$ - такой вещественно-аналитический потенциал на $\mathbb{T}^{2}$, что ни одно из частичных отображений $v\left(\theta_{1}, \cdot\right), v\left(\cdot, \theta_{2}\right)$ не постоянно. Тогда для любого $\varepsilon>0$ существует такое $\lambda_{0}=\lambda_{0}(v, \varepsilon)$, что при $\lambda>\lambda_{0} u \omega \notin \Omega$, где mes $\Omega<\varepsilon$, оператор

$$
H=\lambda v\left(n_{1} \omega_{1}, n_{2} \omega_{2}\right) \delta_{n n^{\prime}}+\Delta
$$

удовлетворяет условию ЛА.

Доказательство этого результата существенно сложнее (заметим, что в теореме 4.2 динамика происходит на $\mathbb{T}$ ). В настоящий момент теорема 1.19 установлена только при $d=2$, и трудность имеет арифметическую природу (контроля за числом решеточных точек на алгебраических поверхностях).

(iv) Динамическая локализация. Это понятие важно в контексте диффузии или отсутствия диффузии в соответствующем уравнении Шрёдингера.

Пусть $H$ - решеточньй оператор Шрёдингера, как и вьше. Рассмотрим соответствующую эволюцию Шрёдингера

$$
i \frac{\partial \psi}{\partial t}=H \psi, \quad \psi(t)=e^{i t H} \psi
$$

где $\psi \in \ell^{2}(\mathbb{Z})$ или $\psi \in \ell^{2}\left(\mathbb{Z}^{d}\right)$. Кроме того, предположим, что $\psi$ удовлетворяет условию убывания

$$
\left|\psi_{n}\right|<|n|^{-A} \text { при }|n| \rightarrow \infty
$$

для некоторого достаточно большого показателя $A$. Понятие “динамической локализации" означает, что

$$
\sup _{t}\left(\sum_{n \in \mathbb{Z}^{d}}\left(1+|n|^{2}\right)\left|\left\langle e^{i t H} \psi, \delta_{n}\right\rangle\right|^{2}\right)^{1 / 2}<\infty,
$$

если $\psi$ удовлетворяет условиям (1.20) и (1.21). Таким образом, это свойство выражает отсутствие “диффузии” и не может вьполняться, если $H$ имеет компоненту непрерьвного спектра (таким образом, условие динамической локализации сильнее условия локализащии). См. также [50].

ТеОрема 1.23. Во всех предьдущих утверждениях, касающихся локализации Андерсона с квазипериодическим потенциалом, имеет место и динамическая локализачия.

Это следует из несколько более аккуратного анализа доказательств. 


\section{2. Решеточные операторы Шрёдингера, связанные с косым сдвигом}

Рассмотрим косой сдвиг на $\mathbb{T}^{2}$ :

$$
T=T_{\omega}: \mathbb{T}^{2} \rightarrow \mathbb{T}^{2}:(x, y) \mapsto(x+y, y+\omega),
$$

с которым мы связьваем решеточньй оператор Шрёдингера на $\mathbb{Z}$,

$$
H=\left(\lambda \cos T^{n}(x, y)\right) \delta_{n n^{\prime}}+\Delta
$$

(при фиксированном $(x, y) \in \mathbb{T}^{2}$ ).

Выражение $\cos T^{n}(x, y)$ мы понимаем как величину $\cos \left(T^{n}(x, y)\right)_{1}$, где

$$
T^{n}(x, y)=\left(x+n y+\frac{n(n-1)}{2} \omega, y+n \omega\right) .
$$

Таким образом, (2.2) - аналог “почти модели Матье”, в которой обычньй сдвиг заменен косым сдвигом. Однако предполагалось (и этому есть некоторые численные подтверждения - W. Schlag, частное сообшение), что спектральное поведение гамильтониана (2.2) совершенно другое, и неясно, верно ли, что это оператор имеет ЛА в типичной ситуации для любого $\lambda>0$ (также и для малых $\lambda$ ). Следовательно, ожидается, что гамильтониан (2.2) ведет себя, как в случайном или сильно перемешиваюшем случае.

К настоящему времени имеется следующий результат, использующий методы теории возмущений.

Tеорема 2.3 [17]. Для любого $\varepsilon>0$ существует такое $\lambda_{0}=\lambda_{0}(\varepsilon)$, что при $\lambda>\lambda_{0}$ найдется такое множество $\Omega \subset \mathbb{T}^{3}$, mes $\Omega<\varepsilon$, что если в (2.2) взять $(\omega, x, y) \notin \Omega$, то Н удовлетворяет ЛА.

Это утверждение тоже остается справедливьм, если $\cos \theta$ заменить любым не постоянным вешественно-аналитическим потенщиалом на $\mathbb{T}$. Кроме того, мы можем зафиксировать $(x, y) \in \mathbb{T}^{2}$ и утверждать наличие ЛА для $\omega \notin \Omega \subset \mathbb{T}$, где mes $\Omega<\varepsilon$. Что касается поведения при малых $\lambda$, то мы можем утверждать следующий факт, подтверждающий высказанную вьше гипотезу.

Теорема 2.4 [5; ч. I]. Для любого $\lambda>0$ существует такое множество $\Omega \subset \mathbb{T}$ положительной мерь, что при $\omega \in \Omega$ гамильтониан $H=\lambda\left(\cos \frac{n(n-1)}{2} \omega\right) \delta_{n n^{\prime}}+\Delta$ имеет некоторый точечный спектр $P$, mes $\bar{P}>0$.

В связи с (1.4), это свойство очевидньм образом отличает сдвиг от косого сдвига.

Матрицы Якоби, связанные с косым сдвигом, естественно возникают в контексте линейных уравнений Шрёдингера. Рассмотрим зависящее от времени уравнение Шрёдингера на $\mathbb{T}=\mathbb{R} / \mathbb{Z}$ :

$$
i \frac{\partial \psi}{\partial t}+a \frac{\partial^{2} \psi}{\partial x^{2}}+i b \frac{\partial \psi}{\partial x}+V(t, x) \psi=0
$$

где $V$ имеет период 1 по $t$ и $x$ и, более точно,

$$
V(t, x)=\kappa(\cos 2 \pi x)\left[\sum_{n \in \mathbb{Z}} \delta(t-n)\right]
$$


$(\delta$ обозначает меру Дирака на $\mathbb{R})$. Таким образом, $V$ соответствует периодической последовательности "ударов". Уравнение (2.5) - так назьваемое "уравнение квантового сингулярно возмущенного ротатора", введенное в [24] и [25] как модель в квантовом хаосе (квантовьй аналог стандартного отображения Чирикова, ср. с [3], [53]). Многие авторы высказывали гипотезу (ср. с [33], [3]), что при типичных значениях параметра для $a, b$ волновая функция $\Psi$, удовлетворяющая уравнению $(2.5),(2.6)$, оказывается почти периодической по времени, и, таким образом, функция $\widehat{\psi(t)}$ (преобразование Фурье) остается локализованной на $\mathbb{Z}$. Эта проблема сводится к динамической локализации для матрицы монодромии $W$ (получаемой сдвигом по времени на единищу, $\psi(0) \rightarrow \psi(1))$. Оператор $W$ унитарен на $L^{2}(\mathbb{T})$ и может быть записан в виде

$$
W=U_{a, b} W_{\kappa}
$$

где

$$
U_{a, b}=\exp \left\{i\left(a \frac{d^{2}}{d x^{2}}+i b \frac{d}{d x}\right)\right\}
$$

$$
W_{\kappa} \text { - оператор умножения на } e^{i \kappa \cos 2 \pi x} \text {. }
$$

При переходе к преобразованиям $\Phi$ урье, $U_{a, b}$ становится диагональньм оператором:

$$
U_{a, b}=e^{i\left(4 \pi^{2} a n^{2}+2 \pi b n\right)} \delta_{m, n}
$$

что объясняет связь с косьм сдвигом.

Для малой константы связи $\kappa$ в (2.6) следующий результат доказьвает гипотезу о почти периодичности.

Tеорема 2.10 [6]. Пусть $\varepsilon>0$. Тогда при фиксированном $b$ в (2.5), при $\kappa<$ $\kappa(\varepsilon)$ и а $\notin$, mes $\Omega<\varepsilon$, поток, определяемый уравнением (2.5), является почти периодическим по времени в следующем смысле.

Если фиксировать показатель Соболева s и предполагать, что $\psi(0) \in H^{s_{1}}(\mathbb{T})$ u $s_{1}$ достаточно велико, то $\psi(t)$ почти периодична как $H^{s}(\mathbb{T})$-значное отображение на $\mathbb{R}$. В частности,

$$
\sup _{t \in \mathbb{R}}\|\psi(t)\|_{H^{s}}<\infty
$$

Доказательство теоремы 2.10 снова использует методы теории возмущений. Подход, не использующий методов теории возмущений, появился в [21]. Имеется такая постоянная $\kappa_{0}$ (допускаюшая явное определение), что при $\kappa<\kappa_{0}$ утверждение теоремы 2.10 вьполняется для почти всех $(a, b)$. Предыдушие результаты показьвают, что “квантовоеподавление хаотической диффузии” имеет место по крайней мере в режиме с малым $\kappa$.

См. также [9], [15] для оценок диффузии в линейных уравнениях Шрёдингера с обшим гладким зависяшим от времени потенциалом (при $D=1$ и $D>1$ ). 


\section{3. КАМ-теория в гамильтоновых дифференциальных уравнениях с частными производными и бесконечномерных системах}

Эта область исследования находится на пересечении дифференциальных уравнений с частными производными и динамических систем, и в последние десятилетия она изучалась несколькими авторами. Общая тема - привлечение методов теории динамических систем в качественной теории гамильтоновых дифференциальных уравнений с частными производными. В этом разделе мы приводим обзор результатов о построении КАМ-торов. В нашей постановке фазовое пространство бесконечномерно, но торы могут быть более низкой (или конечной) размерности. Напомним, что одномерные (соответственно конечномерные, бесконечномерные) инвариантные торы отвечают решению, периодическому (соответственно квазипериодическому, почти периодическому) по времени. Модель может быть либо моделью гамильтонова возмушения линейного уравнения с параметрами, либо возмущением нелинейного интегрируемого уравнения (и в этом случае ищется возможность выделения параметров из нелинейности техникой нормальных форм и амплитудно-частотной модулящии). Бо́льшая часть нашего обсуждения будет связана с первой моделью, и мы тем самьм будем рассматривать нелинейные возмущения линейного уравнения Шрёдингера или волнового уравнения с параметрами. Эта задача исследуется (следуя стандартной КАМ-схеме и распространению теоремы Мельникова на бесконечномерное фазовое пространство) в размерности 1 (с граничным условием Дирихле) в книге С. Б. Куксина [44] (см. также [43]). Мы отсылаем читателя к [43], [44] по поводу полных ссылок, относящихся к достижениям в этом направлении, особенно к работам Вейна по одномерному волновому уравнению. Следуя [44], мы рассмотрим возмущения зависящих от параметра линейных волновых уравнений или уравнений Шрёдингера в размерности 1 таких, так уравнения

$$
\begin{aligned}
u_{t t} & =\left(\frac{\partial^{2}}{\partial x^{2}}-V(x ; a)\right) w-\varepsilon \frac{\partial \varphi}{\partial w}(x, w ; a), \\
i u_{t} & =u_{x x}+V(x ; a) u+\varepsilon \frac{\partial \varphi}{\partial|u|^{2}}\left(x,|u|^{2} ; a\right) u .
\end{aligned}
$$

Здесь $V(x ; a)$ - вещественный периодический гладкий потенщиал, зависящий от $n$ внешних параметров $a=\left(a_{1}, \ldots, a_{n}\right)$. Обозначим через $\left\{\lambda_{j}(a)\right\}$ спектр Дирихле оператора Штурма-Лиувилля $-\frac{d^{2}}{d x^{2}}+V(x ; a)$. Таким образом, $\lambda_{j}(a)=\pi^{2} j^{2}+O(1)$, и мы предполагаем, что вьполнено следующее условие невырожденности:

$$
\operatorname{det}\left\{\partial \lambda_{j}(a) / \partial a_{k} \mid 1 \leqslant j, k \leqslant n\right\} \neq 0
$$

(это условие заменяет классическое условие “кручения"). Если $\left\{\varphi_{j}\right\}$ обозначает соответствующие собственные функции, то $2 n$-мерное линейное пространство

$$
Z^{0}=\text { линейная оболочка }\left\{\varphi_{j}, i \varphi_{j} \mid 1 \leqslant j \leqslant n\right\}
$$

инвариантно относительно потока уравнения (3.2) при $\varepsilon=0$ и расслаивается на инвариантные $n$-торы

$$
\sum_{a, I}^{0}=\left\{\sum_{j=1}^{n}\left(x_{j}^{+}+i x_{n}^{-}\right) \varphi_{j} \mid\left(x_{j}^{+}\right)^{2}+\left(x_{j}^{-}\right)^{2}=2 I_{j}, j=1, \ldots, n\right\},
$$


которые заполнены квазипериодическими решениями уравнения (3.2) при $\varepsilon=0$. Типичньй результат работы [44] состоит в том, что если условие (3.3) вьполнено, то для большинства значений параметра $а$ сушествует инвариантный тор $\sum_{a, I}^{\varepsilon}\left(T^{n}\right)$ вблизи невозмущенного тора $\sum_{a, I}^{0}$, заданного формулой (3.5) и заполненного квазипериодическими решениями уравнения (3.2). Частотньй вектор $\omega_{\varepsilon}$ возмущенного решения $c \varepsilon$-близок к вектору $\omega=\left(\lambda_{1}, \ldots, \lambda_{n}\right)$ невозмущенного решения.

Метод работы [44] не включает одномерного периодического граничного условия, в связи с некоторыми ограничениями метода КАМ (второе условие Мельникова), исключающими кратности в нормальных частотах. Другой подход был инициирован Крейгом и Вейном (см. обзор в [29]) на основе разложения Ляпунова-Шмидта и приводит к периодическому по времени решению возмушенных уравнений при периодических граничных условиях. Этот метод состоит в расшеплении задачи на (конечномерную) резонансную часть ( $Q$-уравнение) и бесконечномерную нерезонансную часть ( $P$-уравнение). В случае дифференциальных уравнений с частньми производными (в отличие от случая конечномерного фазового пространства) возникают проблемы малых знаменателей при решении $P$-уравнений итерационным методом Ньютона, также и в случае периодичности по времени. Записьвая $u$ в виде

$$
u=\sum_{m, k} \widehat{u}(m, k) e^{i m \lambda t} \varphi_{k}(x)
$$

и допуская действие линеаризованного оператора на коэффициентах Фурье $\widehat{u}(m, k)$, получаем операторы вида

$$
\left(m \lambda-\lambda_{k}\right)+\varepsilon T,
$$

где первый член диагонален, а $T$ по сушеству задается тёплицевым оператором с экспоненщиально убьваюшими матричньми элементами. Тогда основная задача состоит в том, чтобы найти разумные границы для операторов, обратных этим операторам.

Заметим, что в то время как при (обычном) KAM-подходе решение гомологических уравнений включает только обрашение диагонального оператора, мы сейчас вынуждены иметь дело с недиагональным оператором. В последующих работах (см. [11], $[7],[4])$ автор развил это разложение Ляпунова-Шмидта для нового доказательства классических теорем КАМ и Мельникова для квазипериодического движения в конечномерном фазовом пространстве, результата в [44] в размерности 1 при периодическом граничном условии (см. [11]), в размерности 2 для нелинейного уравнения Шрёдингера (см. [7]) и, наконец, для нелинейного уравнения Шрёдингера и нелинейного волнового уравнения в любой размерности $D$ (см. [4]). Линеаризованньй оператор, действующий на $\mathbb{Z}^{b+D}$, имеет вид

$$
\left(m_{1} \lambda_{1}+\cdots+m_{b} \lambda_{b}-\mu_{k}\right)+\varepsilon T
$$

где $\lambda=\left(\lambda_{1}, \ldots, \lambda_{b}\right)$ - "тангенциальные" частоты $(b$ - размерность инвариантного тоpa), a $\left(\mu_{k}\right)_{k \in \mathbb{Z}^{D}}$ - "нормальные частоты". Например, в шрёдингеровом случае имеем

$$
\mu_{k} \sim|k|^{2}=k_{1}^{2}+\cdots+k_{D}^{2},
$$

и мы должны столкнуться с проблемой неограниченных (почти) кратностей, как только $D>1$.

Контроль за обратными к операторам вида (3.8) тесно связан с проблемой оценки функций Грина с квазипериодическим потенциалом вида, рассмотренного в разделе 1. 
На самом деле, результаты статьи [4] существенно используют общую технологию теории возмущений (основанную на субгармоничности и теории полуалгебраических множеств), первоначально разработанную для исследования задачи квазипериодической локализации в $\mathbb{Z}^{2}$ (ср. с разделом 1 , (iii)). Эта техника позволяет иметь дело с большими множествами "сингулярных местоположений" (встречаюшимися при $D \geqslant 2$ из-за кратностей), чего трудно достичь обычными методами теории собственных значений.

Сформулируем специальньй результат о стабильности при возмущении зависящих от параметра линейных уравнений, получаемых таким образом.

Рассмотрим постановку задачи для нелинейного уравнения Шрёдингера

$$
i q_{t}=\mathscr{L} q+\varepsilon \frac{\partial H_{1}}{\partial \bar{q}}
$$

где $H_{1}=H_{1}(q, \bar{q})$ - вешественное полиномиальное выражение от $q, \bar{q}$ с вешественными коэффициентами. Здесь $q=\left(q_{n}\right)_{n \in \mathbb{Z}^{d}}$. Выберем конечное множество мод $n_{1}, \ldots, n_{b} \in$ $\mathbb{Z}^{d}$. Оператор $\mathscr{L}$ задается мультипликатором

$$
\left\{\begin{array}{l}
\mu_{n_{j}}=\lambda_{j} \quad(1 \leqslant j \leqslant b), \\
\mu_{n}=|n|^{2} \text { при } n \in \mathbb{Z}^{d} \backslash\left\{n_{1}, \ldots, n_{b}\right\},
\end{array}\right.
$$

где $\lambda=\left(\lambda_{1}, \ldots, \lambda_{b}\right)$ - параметр (меняюшийся в открытом подмножестве $\left.\mathbb{R}^{b}\right)$. Выбор $\mu_{n}=|n|^{2}$ соответствует оператору $-\Delta$ (и для простоты предполагается независимым от $\lambda$ ). Нелинейные задачи без параметров часто могут быть сведены к этому виду путем извлечения параметров из нелинейности и амплитудно-частотной модуляции, как упомянуто вьше.

При $\varepsilon=0$ уравнение (3.10) имеет очевидное квазипериодическое решение

$$
\left\{\begin{array}{l}
q_{n_{j}}(t)=a_{j} e^{i \lambda_{j} t} \quad(1 \leqslant j \leqslant b), \\
q_{n}(t)=0 \text { при } n \notin\left\{n_{1}, \ldots, n_{b}\right\} .
\end{array}\right.
$$

Тогда результат о стабильности (см. [4; гл. ХIX]) утверждает, грубо говоря, что найдутся канторово подмножество $\Omega_{\varepsilon}$ множества $\lambda$-параметров $\Omega, \operatorname{mes}\left(\Omega \backslash \Omega_{\varepsilon}\right) \stackrel{\varepsilon \rightarrow 0}{\rightarrow} 0$, и гладкое отображение $\lambda \mapsto \lambda^{\prime}$ на $\Omega$ такие, что при $\lambda \in \Omega_{\varepsilon}$ существует квазипериодическое решение уравнения (3.10),

$$
q_{n}(t)=\sum_{k \in \mathbb{Z}^{b}} \widehat{q_{n}}(k) e^{i k \cdot \lambda^{\prime} t}
$$

удовлетворяющее условиям

$$
\begin{gathered}
\widehat{q_{n_{j}}}\left(e_{j}\right)=a_{j} \quad(1 \leqslant j \leqslant b) \quad\left(e_{j}-j \text {-й единичньй вектор в } \mathbb{Z}^{b}\right), \\
\left|\widehat{q_{n}}(k)\right| \lesssim e^{-c(|n|+|k|),} \\
\sum_{(n, k) \notin \mathscr{R}}\left|\widehat{q_{n}}(k)\right|<\sqrt{\varepsilon}, \quad \text { где } \mathscr{R}=\left\{\left(n_{j}, e_{j}\right) \mid j=1, \ldots, b\right\} .
\end{gathered}
$$

Соответствуюшая модельная задача для нелинейного волнового уравнения связана с уравнением

$$
i q_{t}=B q+\varepsilon B^{-1} \frac{\partial H_{1}}{\partial \bar{q}}
$$


где $B$ теперь - мультипликатор $\Phi$ урье $\left(\mu_{n}\right)_{n \in \mathbb{Z}^{d}}$, определенньй формулой

$$
\left\{\begin{array}{l}
\mu_{n_{j}}=\lambda_{j}>0 \quad(1 \leqslant j \leqslant b), \\
\mu_{n}=|n|, \text { если } n \notin\left\{n_{1}, \ldots, n_{b}\right\}
\end{array}\right.
$$

(и отвечающий $\sqrt{-\Delta}$ ).

В этой постановке справедлив аналог сформулированной вьше теоремы о стабильности нелинейного волнового уравнения (см. [4; гл. ХХ] ) (в предыдуших работах были исследованы только случаи $b=1$ (решения, периодические по времени) и $D=1$ ).

В несколько другой постановке проблема квазипериодических решений была поднята в работе [34] о неупорядоченных нелинейных динамических системах с близкодействием в качестве взаимодействия (мы также вернемся к этой статье ниже). Типичный пример - уравнение

$$
i \frac{\partial u}{\partial t}=(\varepsilon \Delta+V) u+\delta|u|^{2 p} u \quad\left(p \in \mathbb{Z}_{+}\right),
$$

где $u=\left(q_{i}\right)_{j \in \mathbb{Z}^{d}}, \Delta$ - решеточный лапласиан на $\mathbb{Z}^{D}$, определенный формулой $(1.1)$, и $V=\left(V_{j}\right)_{j \in \mathbb{Z}^{D}}-$ случайньй потенциал. Если $\delta=0$, то мы просто получаем модель Андерсона, и при малом $\varepsilon$ есть динамическая локализация (и, следовательно, почти периодический поток). Если $\delta \neq 0$ в (3.15), то естественные вопросы - это “нелинейные локализации", степень диффузии и т. д. Похоже, что они в основном не решены. Более скромная цель, также поставленная в [34], состоит в построении квазипериодического решения. Это построение было недавно осушествлено в [22], [23] с помошњю описанной выше схемы. Заметим, что диагональная часть линеаризованного оператора становится теперь равной

$$
m_{1} \lambda_{1}+\cdots+m_{b} \lambda_{b}-V_{j} \quad\left(m \in \mathbb{Z}^{b}, j \in \mathbb{Z}^{D}\right),
$$

где $V_{j}$ - независимые одинаково распределенные величины. Ясно, что и здесь следует ожидать появления больших множеств сингулярных местоположений.

Замечательное наблюдение, касаюшееся подхода, использующего разложение Ляпунова-Шмидта, состоит в том, что он не основан априори на гамильтоновой структуре. Действительно, с алгебраической точки зрения единственная проблема в предыдушем изложении связана с реальностью условия на возмущенные частоты $\lambda_{1}^{\prime}, \ldots, \lambda_{b}^{\prime}$ при решении $Q$-уравнений. Однако это условие может оказаться вьполненным также в некоторых других (негамильтоновых) моделях. Например, периодические по времени решения волновых уравнений вида

$$
y_{x x}-y_{t t}+\rho y+\left(y_{t}\right)^{2}=0
$$

были построены в [12].

Хорошо известными примерами интегрируемых гамильтоновых эволюционных уравнений являются

$$
\begin{array}{ll}
i u_{t}+u_{x x} \pm u|u|^{2}=0 & \text { (одномерное кубическое нелинейное } \\
u_{t t}-u_{x x}+\sin u=0 & \text { уравнение Шрёдингера), } \\
u_{t}+u_{x x}+u u_{x}=0 & \text { (уравнение sin-Гордона), } \\
u_{t}+u_{x x x} \pm u^{2} u_{x}=0 & \text { (уравнение Кортевега-де Фриса) }, \\
u_{t}+u_{x x x}+u u_{x}+\partial_{x}^{-1} \partial_{y y} u=0 & \text { (модифицированное уравнение } \\
\text { Кортевега-де } \Phi \text { риса), } \\
\text { (уравнение Кадомцева-Петвиашвили-II) } .
\end{array}
$$


Эти уравнения соответствуют интегрируемым системам в динамическом смысле, и фазовое пространство (здесь нужно быть более точньм) расслаивается на инвариантные торы, заполненные квазипериодическими или почти периодическими решениями. По аналогии с классической теорией, вопрос состоит в устойчивости некоторых из этих решений при малых гамильтоновых возмушениях уравнения. В размерности 1 (заметим, что уравнения (3.18)-(3.21) в действительности одномерны) задача изучалась в ряде работ С.Б. Куксина (ссылки см. в [43]). Конечно, основная идея состоит в том, чтобы извлечь параметры из нелинейности и свести задачу к возмущению линейного уравнения с параметрами. В случае решений с малыми амплитудами оказывается, что этого часто можно достичь несколькими манипуляциями с нормальной формой без привлечения структуры полной интегрируемости уравнения. См. [45] в связи с возмущениями уравнения (3.19) и [11] для построения квазипериодического решения волнового уравнения

$$
u_{t t}-u_{x x}+\rho u+u^{3}+\varepsilon f^{\prime}(u)=0 .
$$

В этом направлении мало что сделано при $D \geqslant 2$. В частности, задача о стабильности при возмущениях уравнения (3.22), по-видимому, не была исследована.

Конечно, проблема выделения достаточно многих параметров из нелинейности может оказаться совершенно нетривиальной и даже главной трудностью задачи. Например, именно так обстоит дело в случае КАМ-теории кеплеровой задачи $N$ тел, движущихся под действием универсальной гравитации (см. [1], [40], [32]).

До сих пор все в нашем обсуждении касалось стабильности конечномерных торов (в бесконечномерном фазовом пространстве). Что можно сказать об инвариантных торах "полной" размерности?

Рассмотрим сначала модели с близкодействием, более точно, модели вида

$$
H(q, \bar{q})=\sum_{j \in \mathbb{Z}} V_{j}\left|q_{j}\right|^{2}+\varepsilon \sum_{j \in \mathbb{Z}}\left(q_{j} \bar{q}_{j+1}+\bar{q}_{j} q_{j+1}\right)+\delta \sum_{j \in \mathbb{Z}} \prod_{k \in S_{j}} q_{k}^{n_{k}} \bar{q}_{k}^{n_{k}^{\prime}} .
$$

Здесь $\left(V_{J}\right)_{j \in \mathbb{Z}}$ снова независимы и одинаково распределены. Одночлены в последнем слагаемом в (3.24) удовлетворяют условию

$$
\begin{gathered}
S_{j} \subset[j-C, j+C], \\
\sum n_{k}=\sum n_{k}^{\prime}, \quad 4 \leqslant \sum\left(n_{k}+n_{k}^{\prime}\right)<C,
\end{gathered}
$$

где $C$ - некоторая постоянная ("близкодействие" связано с предположением (3.25)). (Мы полагаем $D=1$ для простоты.)

Основной результат статьи Фрёлиха-Спенсера-Вейна [34] состоит в построении торов полной размерности для (3.24) при $\varepsilon=0$. Условие этих авторов об убьвании переменных действия было суперэкспоненциальньп, т.е.

$$
I_{j}<e^{-|j|^{1+\tau}} \text { для некоторых } \tau>0 .
$$

Более слабые ограничения были позже предложены в статье Пёшеля [47]; они имеют вид

$$
I_{j}<e^{(\log |j|)^{1+\tau}} \quad \text { для некоторых } \tau>0
$$


(по-видимому, задача остается нерешенной в предположении полиномиального убывания).

И [34], и [47] используют обычную КАМ-схему.

Вернемся к постановке с дифференщиальными уравнениями с частными производными. Выражение нелинейности в моделях Фурье не приводит к близкодействуюшим одночленам. Они имеют общий вид

$$
q_{k_{1}} \bar{q}_{k_{2}} \cdots q_{k_{s-1}} \bar{q}_{k_{s}} \quad \text { c } k_{1}-k_{2}+\cdots+k_{s-1}-k_{s}=0
$$

Если рассматривать одномерный случай нелинейного уравнения Шрёдингера, то резонансные одночлены будут удовлетворять дополнительному соотношению

$$
k_{1}^{2}-k_{2}^{2}+\cdots+k_{s-1}^{2}-k_{s}^{2}=0 .
$$

Предположим, что $k_{1}^{*}=\left|k_{1}\right|$ и $k_{2}^{*}=\left|k_{2}\right|$, где $k_{1}^{*} \geqslant k_{2}^{*} \geqslant \cdots-$ невозрастающая перестановка последовательности $\left(\left|k_{s^{\prime}}\right|\right)_{1 \leqslant s^{\prime} \leqslant s}$.

Из предыдушего следует, что если $k_{1} \neq k_{2}$, то для резонансных одночленов вьполняется соотношение

$$
k_{1}^{*} \leqslant 2\left(k_{3}^{2}+k_{4}^{2}+\cdots\right) \text {. }
$$

На основе этого факта оказьвается, что анализ, проведенный в [34] и [47], может быть проведен и для одномерных нелинейных уравнений Шрёдингера вида

$$
i u_{t}+u_{x x}+M u+\delta u|u|^{2 p}=0 \quad\left(p \in \mathbb{Z}_{+}\right),
$$

где $M u$ - случайньй мультипликатор Фурье, определенный формулой

$$
M u=\sum_{j \in \mathbb{Z}} V_{j} \widehat{u}(j) e^{2 \pi i j x},
$$

a $\left(V_{j}\right)_{j \in \mathbb{Z}}$ независимы и одинаково распределены (см. [13]).

Условия убьвания $I_{j}=\left|u_{j}\right|^{2}$ аналитического типа или типа Жевре действительно достаточны. Более ранние работы по этой проблеме (см. [48], [14]) требовали чрезвычайно быстрых скоростей убьвания (на самом деле неявно).

\section{СПИСОК ЛИТЕРАТУРЫ}

[1] В. И. Арнольд. Малые знаменатели и проблемы устойчивости движения в классической и небесной механике // УМН. 1963. Т. 18. №6. С. 91-192.

[2] S. Aubry, G. André. Analyticity breaking and Anderson localization in incommensurate lattices // Ann. Israel Phys. Soc. 1980. V. 3. P. 133-164.

[3] J. Bellissard. Noncommutative methods in semiclassical analysis // Lecture Notes in Math. 1994. V. 1589. P. 1-64.

[4] J. Bourgain. Green's function estimates for lattice Schrödinger operators and applications // Ann. of Math. Stud. (to appear).

[5] J. Bourgain. On the spectrum of lattice Schrödinger operators with deterministic potential. I, II. Dedicated to the memory of Thomas H. Wolff // J. Anal. Math. 2002. V. 87. P. 37-75; V. 88. P. 221-254.

[6] J. Bourgain. Estimates on Green's functions, localization and the quantum kicked rotor model // Ann. of Math. (2). 2002. V. 156. №1. P. 249-294.

[7] J. Bourgain. Quasi-periodic solutions of Hamiltonian perturbations of 2D linear Schrödinger equations // Ann. of Math. (2). 1998. V. 148. № 2. P. 363-439. 
[8] J. Bourgain. Exposants de Lyapounov pour opérateurs de Schrödinger discrètes quasi-périodiques // C. R. Math. Acad. Sci. Paris. 2002. V. 335. №6. P. 529-531.

[9] J. Bourgain. Growth of Sobolev norms in linear Schrödinger equations with quasi-periodic potential // Comm. Math. Phys. 1999. V. 204. № 1. P. 207-247.

[10] J. Bourgain. Positivity and continuity of the Lyapounov exponent for shifts on $\mathbb{T}^{d}$ with arbitrary frequency vector and real analytic potential // Preprint. Princeton: Institute for Advanced Studies, 2003.

[11] J. Bourgain. Nonlinear Schrödinger equations // Hyperbolic Equations and Frequency Interactions (Park City, UT, 1995). Providence, RI: Amer. Math. Soc., 1999. P. 3-157. (IAS/Park City Math. Ser. V. 5.)

[12] J. Bourgain. Periodic solutions of nonlinear wave equations // Harmonic Analysis and Partial Differential Equations (Chicago, IL, 1996). Chicago: Univ. Chicago Press, 1999. P. 69-97. (Chicago Lectures in Math.)

[13] J. Bourgain. Invariant tori for 1D periodic NLS // Preprint. Princeton: Institute for Advanced Studies, 2003.

[14] J. Bourgain. Construction of approximative and almost periodic solutions of perturbed linear Schrödinger and wave equations // Geom. Funct. Anal. 1996. V. 6. № 2. P. 201-230.

[15] J. Bourgain. On growth of Sobolev norms in linear Schrödinger equations with smooth time dependent potential // J. Anal. Math. 1999. V. 77. P. 315-348.

[16] J. Bourgain, M. Goldstein. On nonperturbative localization with quasi-periodic potential // Ann. of Math. (2). 2000. V. 152. № 3. P. 835-879.

[17] J. Bourgain, M. Goldstein, W. Schlag. Anderson localization for Schrödinger operators on $\mathbb{Z}$ with potentials given by the skew-shift // Comm. Math. Phys. 2001. V. 220. № 3 . P. $583-621$.

[18] J. Bourgain, M. Goldstein, W. Schlag. Anderson localization for Schrödinger operators on $\mathbb{Z}^{2}$ with quasi-periodic potential // Acta Math. 2002. V. 188. № 1. P. 41-86.

[19] J. Bourgain, S. Jitomirskaya. Absolutely continuous spectrum for 1D quasiperiodic operators // Invent. Math. 2002. V. 148. №3. P. 453-463.

[20] J. Bourgain, S. Jitomirskaya. Continuity of the Lyapounov exponent of quasiperiodic operators with analytic potential. Dedicated to David Ruelle and Yasha Sinai on the occasion of their 65th birthdays // J. Statist. Phys. 2002. V. 108. № 5-6. P. 1203-1218.

[21] J. Bourgain, S. Jitomirskaya. Nonperturbative localization for the quantum kicked rotor model // Preprint. Princeton: Institute for Advanced Studies, 2003.

[22] J. Bourgain, M.-W. Wang. Anderson localization for time quasi-periodic random Schrödinger and wave equations // Comm. Math. Phys. (to appear).

[23] J. Bourgain, M.-W. Wang. Quasi-periodic solutions of non-linear random Schrödinger equations // Preprint. Princeton: Institute for Advanced Studies, 2004.

[24] G. Casati, B. V. Chirikov, F. M. Izraelev, J. Ford. Stochastic behavior of a quantum pendulum under a periodic perturbation // Lecture Notes in Phys. 1979. V. 93. P. 334-352.

[25] B. Chirikov, F. Izrailev, D. Shepelyansky. Dynamical stochasticity in classical and quantum mechanics // Soviet Sci. Rev. Sect. C: Math. Phys. Rev. 1981. V. 2. P. 209-267.

[26] V. Chulaevsky, F. Delyon. Purely absolutely continuous spectrum for almost Mathieu operators // J. Statist. Phys. 1989. V. 55. № 5-6. P. 1279-1284.

[27] V.A. Chulaevsky, E. I. Dinaburg. Methods of KAM-theory for long-range quasi-periodic operators on $\mathbb{Z}^{\nu}$. Pure point spectrum // Comm. Math. Phys. 1993. V. 153. №3. P. 559-577.

[28] V. Chulaevsky, Ya. Sinai. Anderson localization for the 1D discrete Schrödinger operator with two-frequency potential // Comm. Math. Phys. 1989. V. 125. № 1. P. 91-121.

[29] W. Craig. Problèmes de petits diviseurs dans les équations aux dérivées partielles. Paris: Société Mathématiques de France, 2000. (Panoramas et Synthèses. V. 9.)

[30] Е. И. Динабург, Я. Г. Синай. Об одном уравнении Шрёдингера с квазипериодическим потенциалом // Функц. анализ и прил. 1975. Т. 9. № 4. С. 8-21.

[31] L. H. Eliasson. Discrete one-dimensional quasi-periodic Schrödinger operators with pure point spectrum // Acta Math. 1997. V. 179. № 2. P. 153-196.

[32] J. Féjoz. Démonstration du "théorème d'Arnold" sur la stabilité du système solaire (d'après M. Herman) // Ergodic Theory Dynam. Systems. (to appear). 
[33] S. Fishman, D. Grempel, R. Prange. Chaos, quantum recurrences, and Anderson localization // Phys. Rev. Lett. 1982. V. 49. № 8. P. 509-512.

[34] J. Fröhlich, T. Spencer, C. E. Wayne. Localization in disordered, nonlinear dynamical systems // J. Statist. Phys. 1986. V. 42. № 3-4. P. 247-274.

[35] J. Fröhlich, T. Spencer, P. Wittwer. Localization for a class of one-dimensional quasi-periodic Schrödinger operators // Comm. Math. Phys. 1990. V. 132. № 1. P. 5-25.

[36] M. Goldstein, W. Schlag. Hölder continuity of the integrated density of states for quasi-periodic Schrödinger operators and averages of shifts of subharmonic function // Ann. of Math. (2). 2001. V. 154. № 1. P. 155-203.

[37] A. Gordon, S. Jitomirskaya, Y. Last, B. Simon. Duality and singular continuous spectrum in the almost Mathieu equation // Acta Math. 1997. V. 178. № 2. P. 169-183.

[38] B. Helffer, J. Sjöstrand. Analyse semi-classique pour l'équation de Harper (avec application à l'équation de Schrödinger avec champ magnétique) // Mém. Soc. Math. France (N.S.). 1988. V. 34.

[39] M. Herman. Une méthode pour minorer les exposants de Lyapounov et quelques exemples montrant le caractère local d'un théorème d'Arnold et de Moser sur le tore de dimension 2 // Comment. Math. Helv. 1983. V. 58. № 3. P. 453-502.

[40] M. Herman. Démonstration d'un théorème de V. I. Arnold // Séminaire de Systèmes Dynamiques. Paris, 1998.

[41] S. Jitomirskaya. Metal-insulator transition for the almost Mathieu operator // Ann. of Math. (2). 1999. V. 150. №3. P. 1159-1175.

[42] А.Н. Колмогоров. О сохранении условно периодических движений при малом изменении функции Гамильтона // Докл. АН СССР. 1954. Т. 98. № 4. С. 527-530.

[43] S. B. Kuksin. Analysis of Hamiltonian PDEs. Oxford: Oxford Univ. Press, 2000. (Oxford Lecture Ser. Math. Appl. V. 19.)

[44] S. B. Kuksin. Nearly Integrable Infinite-dimensional Hamiltonian Systems. Berlin: Springer-Verlag, 1993. (Lecture Notes Math. V. 1556.)

[45] S. B. Kuksin, J. Pöschel. Invariant Cantor manifolds of quasi-periodic oscillations for a nonlinear Schrödinger equation // Ann. of Math. (2). 1996. V. 143. № 1. P. 149-179.

[46] Y. Last. Almost everything about the almost Mathieu operator // XIth International Congress on Mathematical Physics (Paris, 1994). Cambridge: Internat. Press, 1995. P. 366-372.

[47] J. Pöschel. Small divisors with spatial structure in infinite-dimensional Hamiltonian systems // Comm. Math. Phys. 1990. V. 127. № 2. P. 351-393.

[48] J. Pöschel. On the construction of almost periodic solutions for a nonlinear Schrödinger equation // Ergodic Theory Dynam. Systems. 2002. V. 22. № 5. P. 1537-1549.

[49] J. Puig. Cantor spectrum for the almost Mathieu operator // Comm. Math. Phys. 2004. V. 244. № 2. P. 297-309.

[50] R. del Rio, S. Jitomirskaya, Y. Last, B. Simon. Operators with singular continuous spectrum. IV: Hausdorff dimensions, rank one perturbations, and localization // J. Anal. Math. 1996. V. 69. P. 153-200.

[51] B. Simon. Schrödinger operators in the twentieth century // J. Math. Phys. 2000. V. 41. №6. P. 3523-3555.

[52] Ya. G. Sinai. Anderson localization for one-dimensional difference Schrödinger operators with quasi-periodic potential // J. Statist. Phys. 1987. V. 46. № 5-6. P. 861-909.

[53] Ya. G. Sinai. Mathematical problems in the theory of quantum chaos // Lecture Notes in Math. 1991. V. 1469. P. 41-59.

[54] E. Sorets, T. Spencer. Positive Lyapounov exponents for Schrödinger operators with quasi-periodic potential // Comm. Math. Phys. 1991. V. 142. № 3. P. 543-566.

Institute for Advanced Study, Princeton, NJ

Поступила в редакцию

E-mail: bourgain@math.ias.edu

23.01 .2004 